\title{
Louis GILL,
}

Économiste, professeur retraité de l'UQAM

2004

\section{"Notes sur la perspective de la grève générale"}

\author{
Un document produit en version numérique par Jean-Marie Tremblay, bénévole, \\ professeur de sociologie au Cégep de Chicoutimi \\ Courriel:jmt_sociologue@videotron.ca \\ Site web: http://pages.infinit.net/sociojmt
}

Dans le cadre de la collection: "Les classiques des sciences sociales"

Site web: http://www.uqac.uquebec.ca/zone30/Classiques_des_sciences_sociales/index.html

Une collection développée en collaboration avec la Bibliothèque

Paul-Émile-Boulet de l'Université du Québec à Chicoutimi

Site web: http://bibliotheque.uqac.uquebec.ca/index.htm 
Cette édition électronique a été réalisée par Jean-Marie Tremblay, bénévole, professeur de sociologie au Cégep de Chicoutimi à partir de :

Louis Gill, économiste québécois

Professeur retraité de l'UQAM

\section{"Notes sur la perspective de la grève générale"}

Texte rédigé en février 2004 qui n'a pas été publié, mais qui représente un intérêt certain dans le cadre du bras de fer qu'ont commencé à se livrer depuis l'automne dernier, le parti libéral au pouvoir à Québec et de larges couches de la population.

Louis GILL est économiste et professeur retraité du département de sciences économiques de l'UQAM où il a œuvré de 1970 à 2001. Tout au cours de cette carrière, il a eu une activité syndicale active. Il a publié plusieurs ouvrages, sur la théorie économique marxiste, l'économie internationale, l'économie du socialisme, le partenariat social et le néolibéralisme, ainsi que de nombreux essais et articles de revues et de journaux sur des questions économiques, politiques, sociales et syndicales.

[Publication autorisée par M. Gill le 23 juillet 2004.

Courriel : gill.louis@uqam.ca

Polices de caractères utilisée :

Pour le texte: Times, 12 points.

Pour les citations : Times 10 points.

Édition électronique réalisée avec le traitement de textes Microsoft Word 2001 pour Macintosh.

Mise en page sur papier format

LETTRE (US letter), 8.5' x 11'”)

Édition complétée le 19 août 2004 à Chicoutimi, Québec.

\section{Fait avec}

Macintosh 


\section{Table des matières}

\section{La situation}

Quand?

Par qui?

Pour quoi?

Comment? 


\title{
"Notes sur la perspective de la grève générale"
}

\author{
Louis Gill, économiste \\ Professeur retraité, UQAM \\ 3 février 2004
}

\section{La situation}

$\underline{\text { Retour à la table des matières }}$

Le gouvernement Charest a adopté à l'automne 2003 en fin de session, avec une rare arrogance et sous le bâillon, sans mandat et en se réclamant d'un mandat de changement fictif que lui auraient donné les électeurs en avril, un ensemble de lois antisyndicales et antisociales. Comme démonstration de la non-adhésion de la population à ce mandat, un sondage révélait en janvier que $67 \%$ de la population estimait que le parti libéral n'avait pas été élu pour cela. Même au sein du parti libéral, il y aurait de la grogne. À l'automne, en réaction contre l'imminence de l'adoption des projets de loi alors en débat à l'Assemblée nationale, un puissant mouvement de protestation s'est manifesté à travers tout le Québec, mobilisant des centaines de milliers de personnes, syndiquées et non syndiquées, travailleurs, parents protestant contre la hausse des frais de garde, etc. Il a donné lieu à des fermetures des ports, de routes d'importance stratégique, coupant littéralement des régions entières des grands centres, etc. 
Pour diverses raisons, ce mouvement, dont il était prévu au départ qu'il serait seulement ponctuel et ne se prolongerait pas, a effectivement été interrompu et le gouvernement est allé de l'avant. Ses lois sont maintenant adoptées et ont commencé à s'appliquer ou à tout le moins à montrer le type de brèche qu'elles ont ouvert. Déjà, à la faveur de la loi sur la sous-traitance, il est question d'une privatisation partielle des services du transport en commun. Et ce n'est évidemment que la pointe de l'iceberg. Que faire maintenant ? La question de la grève générale a été évoquée par plusieurs. Si on doit recourir à ce moyen, et il semble que ce soit inévitable à moins de capituler, quand, par qui, pour quoi et comment devrait-on y recourir?

\section{Quand?}

La FTQ annonce une vaste consultation de ses membres et précise avec prudence : « la grève générale peut-être, mais pas nécessairement la grève générale ! ». La CSD a elle aussi évoqué la possibilité, alors que des syndicats locaux réclament ouvertement le recours à ce moyen. Il ne fait aucun doute que le temps tarde et qu'il faut agir dans les meilleurs délais. La consultation doit se faire, bien sûr. Mais elle doit se faire à partir d'une proposition claire des directions d'aller dans cette voie, quitte à ce que les membres la rejettent, auquel cas il faudra ultérieurement y revenir lorsque les ravages des lois adoptées se seront révélés sans ambiguïté.

\section{Par qui ?}

Une grève générale est une grève générale. Elle ne saurait être une grève générale de la seule FTQ ou de la seule CSN, etc. Il faut donc tout faire pour surmonter les tensions qui pourraient subsister entre les diverses centrales pour réaliser une unité sans laquelle on peut difficilement espérer gagner.

\section{Pour quoi ?}

Voilà la question clé. L'objectif de l'action à engager doit être le rappel des lois iniques adoptées avant Noël sans mandat de la population, contre la volonté de la majorité par une équipe de destructeurs des institutions de la société québécoise. Se limiter par exemple à dire : «On va avoir l'œil sur ce gouvernement-là, et à la moindre récidive on va le bloquer, si nécessaire par la 
grève générale », est pur verbiage. Nous passerions l'éponge sur l'automne et ferions les fanfarons en promettant au gouvernement de lui livrer une lutte sans merci s'il poursuivait sur sa lancée. Une perspective d'action de ce genre se limiterait à vouloir contrer les seuls effets d'une cause à laquelle on renoncerait de s'attaquer. Admettons, par exemple, qu'une action vigoureuse contre les velléités de privatisation du transport en commun réussissait à bloquer les intentions du gouvernement à cet égard. Quelle garantie cela nous donnerait-il que le gouvernement ne reviendrait pas par la suite à la charge, en s'appuyant sur la même loi qui serait toujours en place? Le président de la société du transport en commune de la ville de Montréal, Claude Dauphin, sentant la fumée, a voulu calmer les esprits en disant : "La privatisation n'est pas pour nous la panacée; en tout cas, ce n'est pas pour demain matin ! » Belle assurance pour après-demain matin, la loi étant là pour le permettre et l'encourager.

\section{Comment?}

D'abord, répétons-le, définissons clairement l'objectif, qui doit être au moins à la hauteur du moyen évoqué, la grève générale. Répéter cela peut sembler superflu et surtout poser le problème à l'envers. Mais justement : le problème est actuellement posé à l'envers. Si on a entendu proposer le moyen d'action qu'est la grève générale, on n'a pas entendu grand chose quant à la déclaration de l'objectif poursuivi. Si on parle de s'engager dans un moyen aussi radical que la grève générale, on a intérêt à savoir pour quoi on veut la faire. Et si on ne veut pas la faire sur la cause, ne nous leurrons pas en prétendant vouloir la faire sur les seuls effets.

Dans les conditions actuelles, le meilleur moyen serait sans doute l'organisation, dans un premier temps, d'une, deux, trois journées de grève générale consécutives, une par semaine, par exemple, en annonçant leur date et en déclarant notre volonté ferme de continuer, tant que le gouvernement n'aura pas rappelé ses lois de l'automne, puis de réévaluer les diverses hypothèses de poursuite immédiate de la lutte, de son report, etc., au terme de ce premier affrontement. Cela veut dire une épreuve politique de première importance. L'objectif qui est le rappel d'un ensemble de lois d'ordre économique et social nous amène d'emblée sur le terrain politique. La question qui est dès lors posée est celle de la survie du gouvernement. Ou il plie et il est, à la limite, forcé de démissionner, parce que tirant le constat de son désaveu par une population dont il prétend depuis le début de se réclamer. 
Ou il ne plie pas et c'est la guerre ouverte. Pour gagner cette bataille, il faut compter sur et s'assurer de l'appui des autres couches de la population qui ne sont pas nécessairement touchées par les lois que nous combattons, mais qui sont mobilisées contre le gouvernement pour d'autres raisons, par exemple contre la construction de la centrale du Suroît, etc. Il faut aussi prévoir les réserves financières qui deviendront rapidement nécessaires.

Il faut tout faire pour gagner cette épreuve de force et partir avec l'idée que le mouvement syndical avec l'appui de larges couches de la population est capable de gagner. Si nous ne faisons rien, nous allons devoir subir les lois de l'automne Le fait qu'elles seront toujours en place et, plus encore, notre éventuelle inaction seront pour le gouvernement de puissants points d'appui sur lesquels il pourra s'appuyer pour poursuivre sur sa lancée dans l'avenir. Si nous ne voulons pas donner l'impression de vouloir combattre un tigre avec un tue-mouches, il faut agir. À l'inverse, brandir l'arme de la grève générale, mais laisser en place les lois votées équivaudrait à vouloir laisser le tigre bien vivant en prétendant frapper avec une masse les mouches qui tournent autour.

Nous pouvons gagner si le mouvement est fort. Il faut par contre, cela va de soi, ne pas écarter la possibilité de perdre. Il n'y a jamais d'assurance à cet égard au point de départ. Le gouvernement peut résister, recourir à la répression. Il faut être conscients que cela peut se produire, mais que même dans cette hypothèse nous pouvons remporter des gains réels, sur le plan politique, qui pourront porter leurs fruits plus tard. À travers ce mouvement peut se construire une force politique organisée des travailleurs. D'autres luttes de ce genre ont été livrées par les travailleurs dans le passé. Certaines ont été victorieuses, d'autres ont été perdues. Après de multiples grèves, générales, de durée indéterminée, le mouvement ouvrier brésilien, par exemple, s'est construit syndicalement, avec la CUT (Centrale unique des travailleurs), et politiquement, avec le PT (Parti des travailleurs). Parfois, la victoire ne se mesure pas uniquement par rapport à l'atteinte immédiate des objectifs précis qui étaient à l'origine du déclenchement de la bataille. Celui qui est aujourd'hui président du Brésil, Lula, dont l'action qu'il a dirigée, d'abord sous la dictature militaire, a été des plus dures et dont les objectifs immédiats de nature économique ont souvent été déçus, tire aujourd'hui le bilan positif suivant de cette action : «Nous avons peu gagné en ce qui a trait par exemple à nos revendications salariales, mais nous avons remporté des gains beaucoup plus importants, contre la répression, les emprisonnements, etc., qui sont d'une autre nature: nous avons construit la CUT et le PT et aujourd'hui nous avons conquis le pouvoir au niveau du pays ». 
Il va sans dire que cette question-là ne peut aussi que se poser dans une bataille impliquant une grève générale dont les incidences sont inévitablement d'ordre politique. Si le gouvernement Charest, par exemple, devait démissionner sous la force du mouvement de revendication que nous voulons construire et dont le contenu est éminemment politique, elle ne manquerait pas de se poser au moment de le remplacer par un autre gouvernement.

Fin du texte. 